\title{
Wind beyond the tip of the AGB and its relevance to stardust grain data
}

\section{J. Buntain ${ }^{* \dagger}$}

Centre for Stellar and Planetary Astrophysics - Monash University, Victoria, Australia

E-mail: Joelene.Buntain@monash.edu

\section{Lugaro}

Centre for Stellar and Planetary Astrophyics - Monash University, Victoria, Australia

E-mail: Maria.Lugaro@monash.edu

\section{R. J. Stancliffe}

Centre for Stellar and Planetary Astrophyics - Monash University, Victoria, Australia

E-mail: Richard.Stancliffe@monash.edu

\section{A. Karakas}

Mount Stromlo Observatory - Australian National University, Canberra, Australia

E-mail: akarakas@mso.anu.edu.au

L. Nittler

Carnegie Institute of Washington, USA

E-mail: lnittlerdciw.edu

P. Hoppe

Max-Planck-Institute for Chemistry - Mainz, Germany

E-mail: peter.hoppe@mpic.de

\begin{abstract}
An asymptotic giant branch (AGB) star evolves to the post-AGB phase and then may become the core of a planetary nebula. The star evolves at a constant luminosity to hotter temperatures while the mass of the thin H-rich region decreases because of winds. Stardust oxide and silicate grains are formed around AGB stars and recovered from meteorites. The origin of the "Group II grains" is currently explained by invoking the occurence of some kind of extra-mixing process in AGB stars. We propose an alternative explanation: these grains may have originated from the winds of post-AGB stars and planetary nebula cores. We predict that this wind shows the signature of $\mathrm{H}-$ burning. We compare our predictions from stellar models to the compositions observed in Group II stardust oxide and silicate grains to assess this hypothesis. We find that the $\mathrm{O}$ - and Al-isotopic composition of the winds is very close to that of Group II grains, especially if some mixing of the ejected material is performed. However, the Mg-isotopic composition of the winds does not provide as close a match to the Group II grains.
\end{abstract}

11th Symposium on Nuclei in the Cosmos, NIC XI

July 19-23, 2010

Heidelberg, Germany

\footnotetext{
*Speaker.

${ }^{\dagger}$ A footnote may follow.
} 


\section{Introduction}

Stardust grains are recovered from primitive meteorites and analysed in the laboratory (Fig. 1) [1]. Stardust and oxide silicate grains are classified into four groups [2]: Group I grains show exesses in ${ }^{17} \mathrm{O}$ and are believed to have originated from red giants and AGB stars. Group II grains show deficits in ${ }^{18} \mathrm{O}$ and enrichments in ${ }^{17} \mathrm{O}$ and are currently explained by the presence of extramixing in AGB stars. Group III grains show deficits in ${ }^{17} \mathrm{O}$ and ${ }^{18} \mathrm{O}$ and their origin is unclear. Group IV grains show excesses in ${ }^{18} \mathrm{O}$ and are believed to have originated in supernovae. Focusing on Group II grains, the current explanation for their composition involves the presence of some kind of extra-mixing process in AGB stars [3]. The physical mechanism responsible for such deep mixing remains unclear (even though work is in progress on this problem $[4,5]$ ) and it is debated if this mechanism is really needed to explain stellar observations [6,7].

In this paper we wish to test an alternative explanation for the composition of oxide and silicate stardust grains of Group II by linking their origin to the wind episodes that occur beyond the tip of the AGB. After its $\mathrm{H}$-rich envelope is reduced to $\simeq 10^{-2} \mathrm{M}_{\odot}$, an asymptotic giant branch (AGB) star evolves to become a post-AGB star and then may become the core of a planetary nebula. The star evolves at a constant luminosity to hotter temperatures while the mass of the thin H-rich region decreases because of winds. These winds carry the signature of the H-burning material that gets progressively exposed to the stellar surface.

\section{Methods and results}

To look at the composition of post-AGB and planetary nebula core winds we took a $1.25 \mathrm{M}_{\odot}$ model of solar metallicity from [9] and selected the deepest $10^{-3} \mathrm{M}_{\odot} \mathrm{H}$-rich region of the star during a typical interpulse period. The material has $\mathrm{C} / \mathrm{O}<1$ because we have considered a stellar model that does not experience any dredge-up of material from the He- and C-rich region into the envelope during its AGB phase. We simulated mixing of the ejected material, as it may occur in the winds or during the interaction of the winds with the surrounding material. We experimented with different types of mixing possibilities. We refer the reader to [8] for a detailed description of these different mixing schemes. In Figs. 3, 4, 5 and 6 we present the O-, Al- and Mg-isotopic compositions obtained by keeping the material stratified ("unmixed") or mixed in the different ways described in [8] and compare them to the stardust data points.

Figs. 3 and 4 show that the "unmixed" compositions (long-short dashed line) do not provide a very close match to the Group II grains. The "mixed" compositions (solid lines) allowed us to move away from the solar composition to reach the majority of the Group II grains and to cover the ${ }^{18} \mathrm{O} /{ }^{16} \mathrm{O}$ and ${ }^{26} \mathrm{Al} /{ }^{27} \mathrm{Al}$ grain data, except that the ${ }^{17} \mathrm{O} /{ }^{16} \mathrm{O}$ ratios are not matched since they remain roughly $50 \%$ higher than the data points. To match the Group II grains, our results show that we need to mix within the inner half of the total $10^{-3} \mathrm{M}_{\odot}$ mass we are considering. If we include in the mixture too much material from the outer half of the mass range, we quickly move towards ${ }^{18} \mathrm{O} /{ }^{16} \mathrm{O}$ ratios greater than 0.001 . The different mixing schemes for the $\mathrm{Mg}$ compositions (Figs. 5 and 6) were not as successful in covering the data. They appear to be able to cover the negative but not the positive $\delta^{25} \mathrm{Mg} /{ }^{24} \mathrm{Mg}$ values. ${ }^{25} \mathrm{Mg}$ can be enhanced in the He-rich layer located just below the region we are considering here due to ${ }^{22} \mathrm{Ne}(\alpha, \mathrm{n}){ }^{25} \mathrm{Mg}$ reactions, however, mixing material 


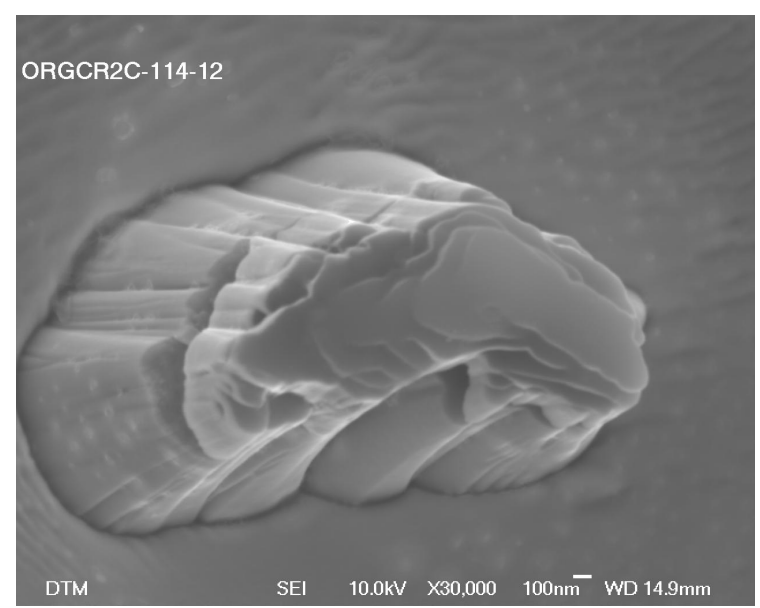

Figure 1: Corundum $\left(\mathrm{Al}_{2} \mathrm{O}_{3}\right)$ stardust grain recovered from a primitive meteorite. The size of this grain is approximately $2.3 \mu \mathrm{m}$.

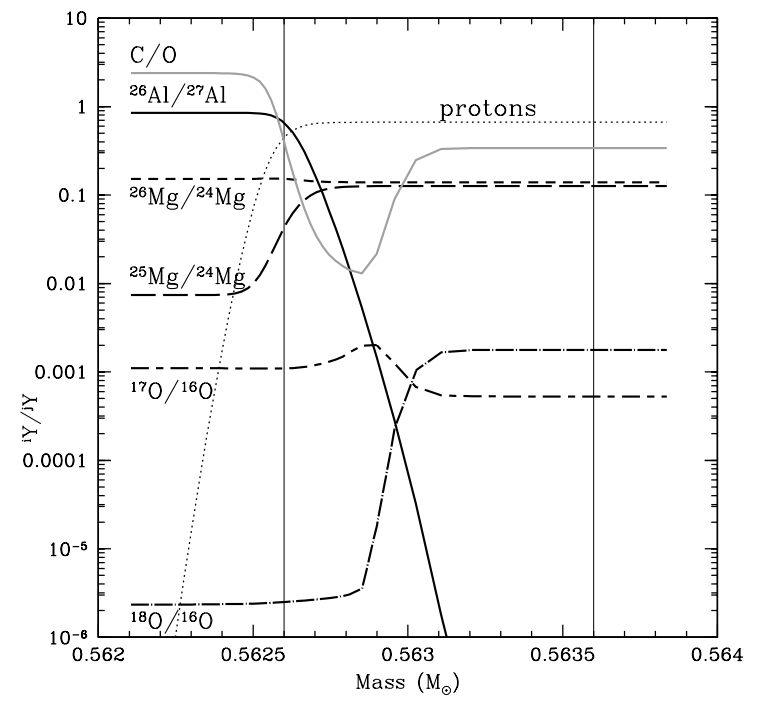

Figure 2: Isotopic ratios in a 1.25 solar mass AGB star with $\mathrm{Z}=0.02$ as a function of the mass coordinate, where mass $=0$ is the centre of the star. The vertical black lines enclose the deepest $10^{-3} \mathrm{M}_{\odot} \mathrm{H}$-rich layer during a typical interpulse period. We assume that this material is representative of the material lost in the winds after the tip of the AGB. The dotted line represents the abundance of protons and helps locate the underlying H-burning shell at roughly $0.5625 \mathrm{M}_{\odot}$. These compositions clearly show the typical signature of H-burning.

from this region would produce C-rich compositions, while oxide grains need O-rich conditions to form.

\section{Discussion}

The main problem of our proposed scenario is that the total mass involved in the grain formation process is very small. Since the mass of the AGB envelope lost during AGB winds, where the vast majority of oxide and silicate grains form, is $\simeq 0.7 \mathrm{M}_{\odot}$, the mass considered here represents less than $0.1 \%$ of the total mass lost in the stellar winds. On the other hand, approximately $10-15 \%$ of presolar oxides and silicate grains belong to Group II. So how can we produce such a large proportion of grains from such a small fraction of mass?

One possibility for dust formation in our scenario are shocks in the post-AGB winds, which dynamically interact with the material that exists around the central star from the previous AGB wind episodes (eg. [10]). Dust is observed to form in post-shock regions in different environments, from the colliding winds of binary massive stars like Eta Carinae and Wolf Rayet stars of WC type, to supernovae post-shock regions (see [11] and references therein). The scenario involving extramixing in AGB stars is much more accurate in reproducing the data [12], but our alternative cannot be excluded for the moment, while its unknown if dust is formed from the winds from central stars of planetary nebulae. To our knowledge, there are no theoretical or observational studies about 


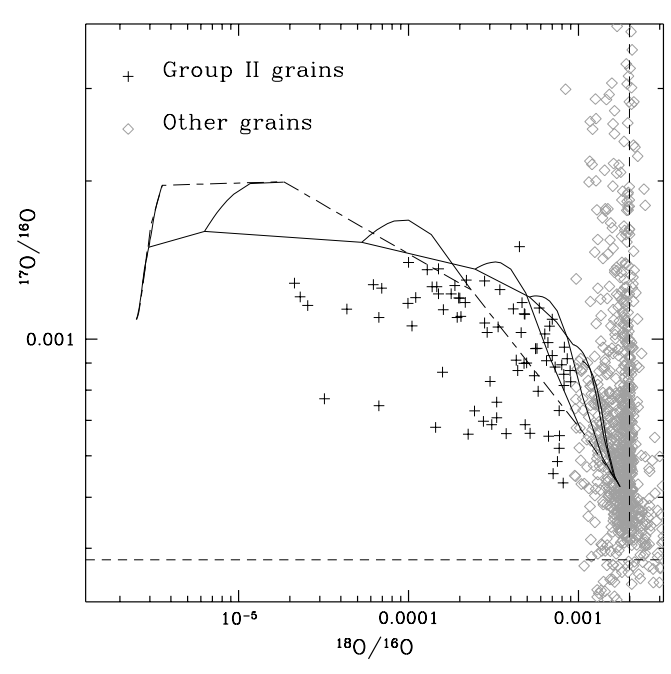

Figure 3: The ${ }^{17} \mathrm{O} /{ }^{16} \mathrm{O}$ versus ${ }^{18} \mathrm{O} /{ }^{16} \mathrm{O}$ isotopic ratios corresponding to the stratified composition ("unmixed", long-short dashed line) and to the mixed composition (solid lines) from different mixing schemes [8], compared to data for oxide and silicate stardust grains. Vertical and horizontal dashed lines represent the solar value.

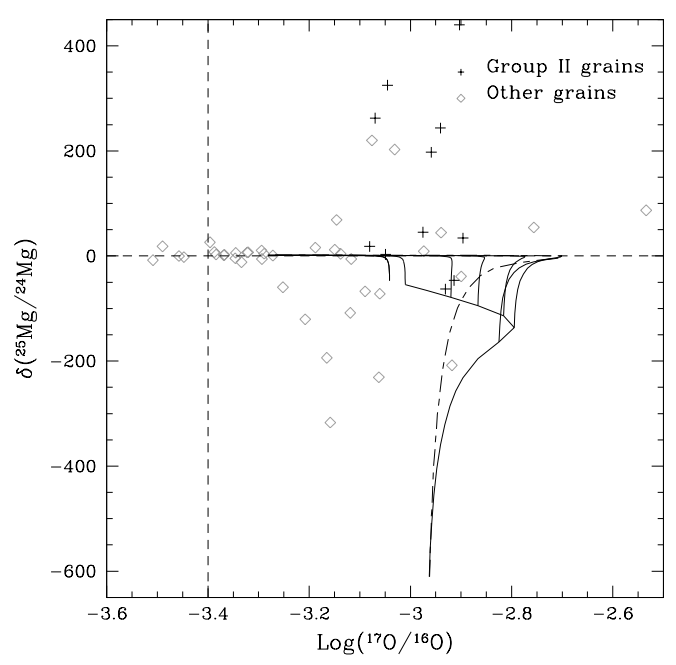

Figure 5: ${ }^{25} \mathrm{Mg} /{ }^{24} \mathrm{Mg}$ versus ${ }^{17} \mathrm{O} /{ }^{16} \mathrm{O}$ in presolar grains and from our model predictions. The lines are the same as in Figs 3 and $4 .{ }^{25} \mathrm{Mg} /{ }^{24} \mathrm{Mg}$ are given as $\delta$-values, which represent permil variations with respect to solar: $\delta^{25} \mathrm{Mg} /{ }^{24} \mathrm{Mg}=$ $\left\{\left[\left(\left({ }^{25} \mathrm{Mg} /{ }^{24} \mathrm{Mg}\right) /\left({ }^{25} \mathrm{Mg} /{ }^{24} \mathrm{Mg}\right) \odot-1\right) \times 1000\right]\right\}$

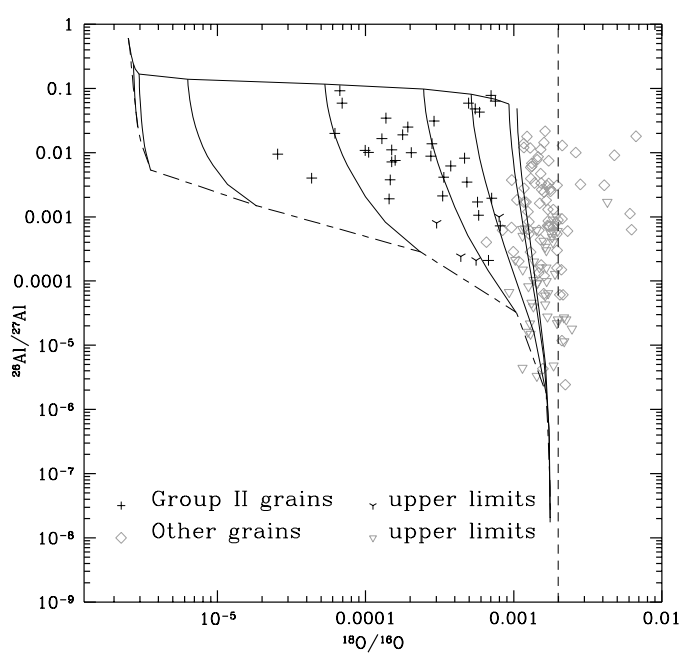

Figure 4: Same as Fig. 3 but for the ${ }^{26} \mathrm{Al} /{ }^{27} \mathrm{Al}$ versus ${ }^{18} \mathrm{O} /{ }^{16} \mathrm{O}$ isotopic ratios.

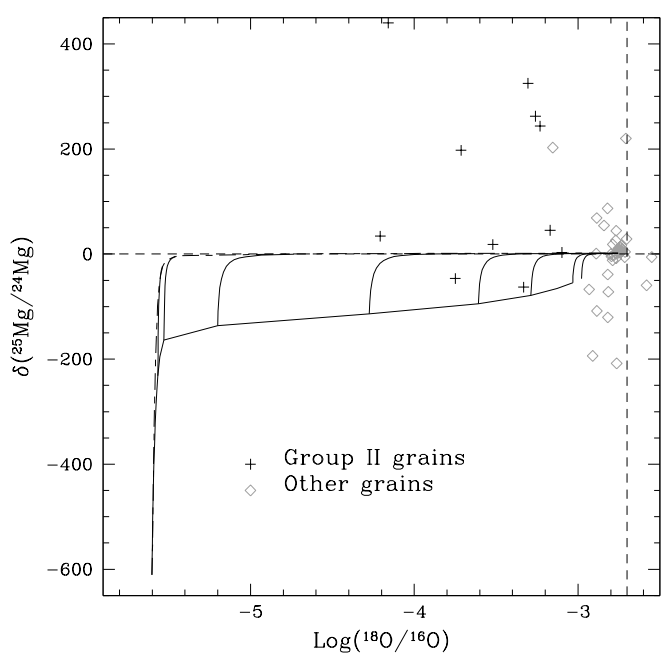

Figure 6: Same as Fig. 5 but for the $\delta^{25} \mathrm{Mg} /{ }^{24} \mathrm{Mg}$ versus ${ }^{18} \mathrm{O} /{ }^{16} \mathrm{O}$. 
the possibility of grain formation in post-shock regions in planetary nebulae, and does about the resulting grain size distribution and features. Also, we need to perform a close revision of the reaction rates we are using before any conclusions can be made.

\section{References}

[1] D. D. Clayton and L. R. Nittler, Astrophysics with Presolar Stardust, ARA\&A 42 (Sept., 2004) 39-78.

[2] L. R. Nittler, C. M. O. Alexander, X. Gao, R. M. Walker, and E. Zinner, Stellar Sapphires: The Properties and Origins of Presolar AL2O3 in Meteorites, ApJ 483 (July, 1997) 475-495.

[3] K. M. Nollett, M. Busso, and G. J. Wasserburg, Cool Bottom Processes on the Thermally Pulsing Asymptotic Giant Branch and the Isotopic Composition of Circumstellar Dust Grains, ApJ 582 (Jan., 2003) 1036-1058, [arXiv: astro-ph/0211271].

[4] M. Busso, G. J. Wasserburg, K. M. Nollett, and A. Calandra, Can Extra Mixing in RGB and AGB Stars Be Attributed to Magnetic Mechanisms?, ApJ 671 (Dec., 2007) 802-810, [0708.2949].

[5] P. A. Denissenkov, M. Pinsonneault, and K. B. MacGregor, Magneto-Thermohaline Mixing in Red Giants, ApJ 696 (May, 2009) 1823-1833, [0 806.4346$].$

[6] A. I. Karakas, S. W. Campbell, and R. J. Stancliffe, Is Extra Mixing Really Needed in Asymptotic Giant Branch Stars?, ApJ 713 (Apr., 2010) 374-382, [1 002 . 4904 ].

[7] M. Busso, S. Palmerini, E. Maiorca, S. Cristallo, O. Straniero, C. Abia, R. Gallino, and M. La Cognata, On the Need for Deep-mixing in Asymptotic Giant Branch Stars of Low Mass, ApJ 717 (July, 2010) L47-L51, [1005.3549].

[8] J. Buntain, M. Lugaro, R. J. Stancliffe, A. Karakas, L. R. Nittler, and P. Hoppe, Wind beyond the tip of the AGB and its relevance to stardust grain data, MemSaIt (July, 2010) in press.

[9] A. Karakas and J. C. Lattanzio, Stellar Models and Yields of Asymptotic Giant Branch Stars, PASA 24 (Oct., 2007) 103-117, [0 708 . 4385].

[10] M. Grewing, Wind features and wind velocities., in Planetary Nebulae (S. Torres-Peimbert, ed.), IAU Symposium 131, pp. 241-250, 1989.

[11] N. Smith, Episodic post-shock dust formation in the colliding winds of Eta Carinae, MNRAS 402 (Feb., 2010) 145-151, [0 910 . 4395].

[12] S. Palmerini, M. La Cognata, S. Cristallo, and M. Busso, Deep Mixing in Evolved Stars. I. The Effect of Reaction Rate Revisions from C to Al, ApJ 729 (Mar., 2011) 3-+, [1011.3948]. 